\title{
Prestimulus Oscillatory Activity over Motor Cortex Reflects Perceptual Expectations
}

\author{
Floris P. de Lange, ${ }^{1}$ Dobromir A. Rahnev, ${ }^{1,2}$ Tobias H. Donner, ${ }^{3 \star}$ and Hakwan Lau ${ }^{1,2 \star}$ \\ ${ }^{1}$ Radboud University Nijmegen, Donders Institute for Brain, Cognition and Behavior, $6500 \mathrm{HB}$, Nijmegen, The Netherlands, ${ }^{2}$ Columbia University, \\ Department of Psychology, New York, New York 10027, and ${ }^{3}$ Department of Psychology, University of Amsterdam, 1012 ZA, Amsterdam, The Netherlands
}

When perceptual decisions are coupled to a specific effector, preparatory motor cortical activity may provide a window into the dynamics of the perceptual choice. Specifically, previous studies have observed a buildup of choice-selective activity in motor regions over time reflecting the integrated sensory evidence provided by visual cortex. Here we ask how this choice-selective motor activity is modified by prior expectation during a visual motion discrimination task. Computational models of decision making formalize decisions as the accumulation of evidence from a starting point to a decision bound. Within this framework, expectation could change the starting point, rate of accumulation, or the decision bound. Using magneto-encephalography in human observers, we specifically tested for changes in the starting point in choice-selective oscillatory activity over motor cortex. Inducing prior expectation about motion direction biased subjects' perceptual judgments as well as the choice-selective motor activity in the $8-30 \mathrm{~Hz}$ frequency range before stimulus onset; the individual strength of these behavioral and neural biases were correlated across subjects. In the absence of explicit expectation cues, spontaneous biases in choice-selective activity were evident over motor cortex. These also predicted eventual perceptual choice and were, at least in part, induced by the choice on the previous trial. We conclude that both endogenous and explicitly induced perceptual expectations bias the starting point of decision-related activity, before the accumulation of sensory evidence.

\section{Introduction}

Prior beliefs about the visual world can strongly bias perception and visually guided behavior. For example, statistical regularities of observed motion direction alter the perception of new motion directions, inducing an attractive bias in the perceived direction (Chalk et al., 2010). It is, however, largely unclear how and at what processing stages these biases affect the perceptual decision.

Perceptual decisions can be formalized as a process of the accumulation of sensory evidence toward a decision bound (Usher and McClelland, 2001; Smith and Ratcliff, 2004; Bogacz et al., 2006; Ratcliff and McKoon, 2008). Within this scheme, prior beliefs could impact the decision at several stages.

First, expectation could alter the state of the decision variable already before the onset of accumulation of sensory evidence. Second, expectation could change the gain (or rate) of accumulation toward the expected outcome. Third, expectation could selectively lower the amount of evidence required for the expected outcome (i.e., the decision bound). In this study, we set

\footnotetext{
Received March 6, 2012; revised Nov. 21, 2012; accepted Nov. 22, 2012.

Author contributions: F.P.d.L. and H.L. designed research; F.P.d.L. and D.A.R. performed research; F.P.d.L., D.A.R., and T.H.D. analyzed data; F.P.d.L., D.A.R., T.H.D., and H.L. wrote the paper.

H.L. and F.P.d.L. received funding from the Human Frontiers Science Project (HFSP Short-Term Fellowship), the Netherlands Organisation for Scientific Research (NWO-VENI and NWO-Open Competition), and the Templeton Foundation (Grant 21569)

*T.H.D. and H.L. contributed equally to this work.

Correspondence should be addressed to Floris P. de Lange, Donders Institute for Brain, Cognition and Behavior, Radboud University Nijmegen, Kapittelweg 29, 6500 HB Nijmegen, The Netherlands. E-mail: floris.delange@donders.ru.nl.

DOI:10.1523/JNEUROSCI.1094-12.2013

Copyright (C) 2013 the authors $\quad 0270-6474 / 13 / 331400-11 \$ 15.00 / 0$
}

out to test whether expectation changes the starting point of choice-selective neural activity.

When decisions are coupled to an effector, previous studies have shown that the ongoing decision process may be "read out" by monitoring activity in neural structures that are involved in preparing the associated motor response (Gold and Shadlen, 2007; Heekeren et al., 2008; Donner et al., 2009; Gould et al., 2012). In monkeys, such accumulation processes have been extensively documented in oculomotor regions of parietal and prefrontal cortex, when perceptual decisions were coupled to saccadic eye movements (Shadlen and Newsome, 1996; Schall, 2001; Yang and Shadlen, 2007). Similarly, recent studies in humans have observed evidence of accumulation-related activity in response-related regions when the perceptual decision was coupled to specific effectors (Tosoni et al., 2008; Donner et al., 2009; Gould et al., 2012), in line with the proposition that there is substantial "leakage" of perceptual and cognitive states into the motor system (Song and Nakayama, 2009). Therefore, motor activity could potentially provide a window into the dynamics of the perceptual decision process.

Using these neuronal markers, neurophysiological and neuroimaging studies have garnered some empirical support for both changes in starting point (Shadlen and Newsome, 2001; Churchland et al., 2008; Preuschhof et al., 2010; Rao et al., 2012) and sensory gain (Rahnev et al., 2011) as a result of biases and learning, but it remains an open question how perceptual decision making is altered by explicitly inducing prior expectations.

Here we examine this issue by investigating how activity in choice-selective motor activity before stimulus onset is affected by perceptual expectations. Subjects made decisions about the 
A left $>$ right choice

[60 90] Hz, [-250 0] ms pre-choice

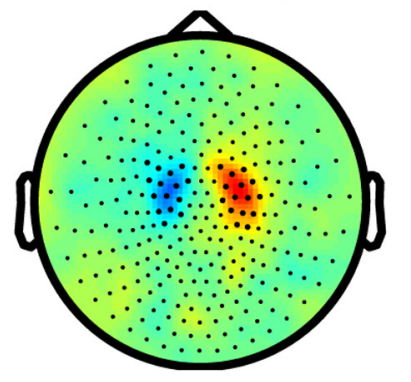

left $>$ right choice

[830] $\mathrm{Hz}$, [-250 0] ms pre-choice

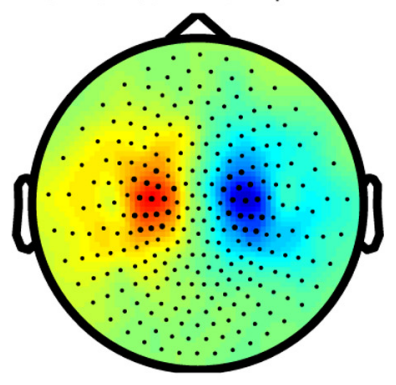

C expect left $>$ right

[7 34] $\mathrm{Hz},[-600 \mathrm{0}$ ] ms pre-stimulus

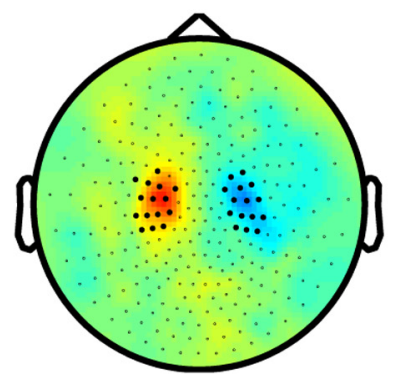

B

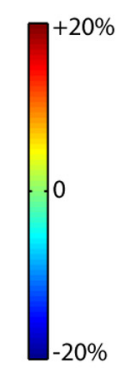

$20 \%$

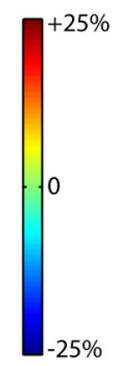

$-25 \%$

\section{D}

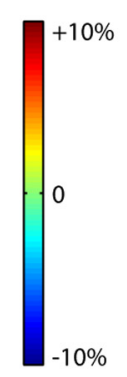

stimulus $>$ baseline

[60 140] Hz, [200 500] ms post-stimulus

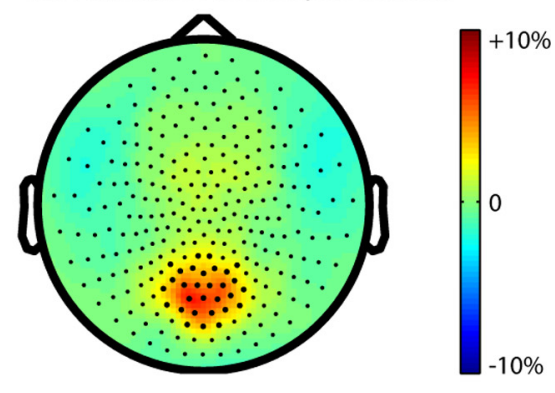

stimulus $>$ baseline

[8 30] Hz, [200 500] ms post-stimulus

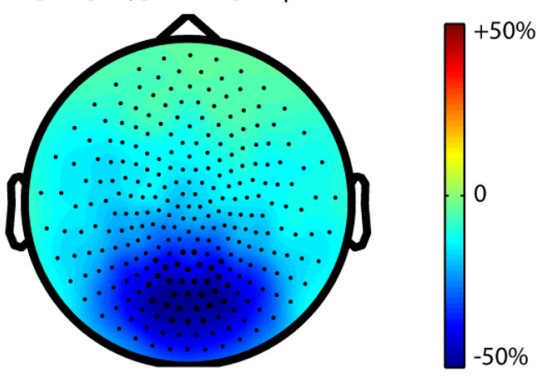

$-50 \%$
[7 15] Hz, [-1050 -100] ms pre-stimulus

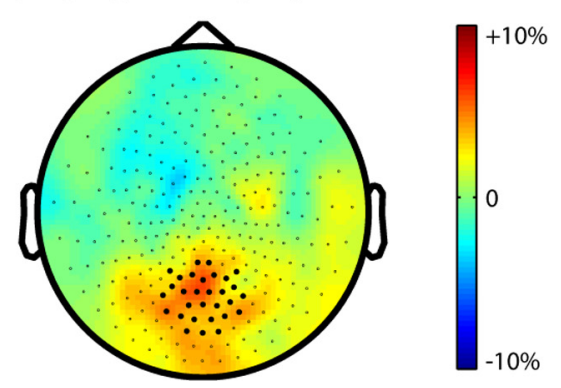

approved by the local ethics committee at Radboud University Nijmegen, and a written informed consent was obtained from the subjects according to the Declaration of Helsinki.

Stimuli. Target stimuli were composed of white dots $\left(\right.$ density $=2.4$ dots $/{ }^{\circ 2} ;$ speed $=6 \%$ s $)$ presented on a black annulus (outer circle radius $=10^{\circ}$; inner circle radius $=1^{\circ}$ ). Stimuli were projected from a calibrated LCD projector (situated outside the magnetically shielded room) onto a back-projection screen through a tube by a mirror system. The refresh rate of the projector was $60 \mathrm{~Hz}$. The direction of net motion was either leftward or rightward. For each frame, a random subset of the dots was chosen to carry the coherent motion. Incoherent dots moved randomly with the same speed as the coherent dots. When dots moved off the annulus, they were replotted at a random location within the annulus. We used three levels of motion coherence (low, intermediate, high). The intermediate coherence level was individually chosen to produce $\sim 75 \%$ correct responses in the absence of a cue (mean proportion of coherently moving dots $=11.1 \%, \mathrm{SD}=3.3 \%$, range $=4.8-18.4 \%)$. The low motion coherence was fixed at $50 \%$ of the intermediate coherence (mean proportion of coherently moving dots $=5.5 \%$ ), while the high motion coherence was $200 \%$ of the intermediate coherence (mean proportion of coherently moving dots $=22.2 \%$ ).

A small fixation square was presented for the duration of the trial, and subjects were required to maintain fixation on it. The stimuli were presented on a gray background and were generated using Psychophysics Toolbox (Brainard, 1997) in MATLAB (MathWorks).

Experimental design. Each trial began with the presentation of a cue. The cue was either predictive ("left" or "right," each $33 \%$ of all trials) or nonpredictive ("neutral," $33 \%$ of all trials) for the upcoming net direction of stimulus motion. The predictive cues correctly indicated the upcoming motion direction on $75 \%$ of the trials. We refer to these trials as "valid." In the remaining $25 \%$ of the trials, the predictive cues indicated the wrong direction of motion. We refer to these trials as "invalid," meaning invalidly cued. The nonpredictive cues were followed by leftward and rightward motion equally often. The nonpredictive cues are referred to as neutral in the rest of the pa-

direction of motion in moving random-dot displays. We induced perceptual expectations by providing probabilistic cues about motion direction and tracked the effect on the decision variable by coupling different perceptual decisions to different motor responses and measuring motor cortical activity before and during the perceptual decision. We also investigated whether spontaneous fluctuations in prestimulus motor cortical activity could affect perceptual choice, in the absence of explicit expectation cues, and we sought to link these spontaneous biases to sequential effects of choice behavior over trials (Gao et al., 2009).

\section{Materials and Methods}

Participants. Nineteen healthy participants (12 male, 7 female; age, $25 \pm$ 4.7 years, mean $\pm S D$ ) participated in the experiment. None of the participants had a history of neurological or psychiatric disorders. All participants had normal or corrected-to-normal vision. The study was per. Thus, the optimal strategy in this task (in terms of maximizing percentage correct) was to apply a directional bias corresponding to the cue (i.e., zero bias for neutral cues). Subjects were fully informed about the above contingencies and were encouraged to take the cue into account when making their perceptual decisions.

The cue consisted of one of the words left, right, or neutral that was presented for $200 \mathrm{~ms}$ followed by a fixation dot that lasted between 800 and $1300 \mathrm{~ms}$. Then, the motion stimulus appeared until subjects provided their response or $3 \mathrm{~s}$ had elapsed. Subjects responded with the index finger of their left hand when they perceived leftward motion and with the index finger of their right hand when they perceived rightward motion. After the response, there was an intertrial interval of between 2 and $2.5 \mathrm{~s}$.

Before the magneto-encephalography (MEG) experiment, each subject took part in a $30 \mathrm{~min}$ training session, in which the subject performed 138 trials, distributed over six training blocks, to establish motion coher- 
ence thresholds (see Stimuli). The first three training blocks (in a total of 30 trials) had a fixed and high coherence to get subjects acquainted with the task demands. Then, based on the performance on the third block (of 10 trials), the coherence level was chosen for the fourth block ( 24 trials), and so on for the fifth (24 trials) and the final sixth (60 trials) block. This method of thresholding ensured a reasonably stable performance for the medium coherence $(70.0 \pm 12.8 \%$, mean $\pm \mathrm{SD})$, within the time constraints posed by the experiment. During the experiment, the presentation of motion coherence was pseudo-randomized for each subject such that each coherence level appeared equally often with each combination of cue identity and motion direction. The experiment consisted of six blocks of 144 trials, with predictive and nonpredictive trials randomly intermixed.

Behavioral analysis. We computed reaction times and error rates, as well as the signal detection theoretic measures $d^{\prime}$ and $c$ (Green and Swets, 1966; Macmillan and Creelman, 2005). $d^{\prime}$ quantifies a subject's stimulus sensitivity (in signal-to-noise ratio units), whereas $c$ quantifies a subject's decision criterion (a nonzero $c$ value implies a bias). These measures were calculated on the basis of the proportion of leftward choices on leftward and rightward motion trials (analogous to hits and false alarms in a detection task). To quantify the effect of the cue on sensitivity and criterion, we computed $d^{\prime}$ and $c$ separately for trials preceded by left, right, or neutral cues. We refer to these measures for the left cue trials as $d^{\prime}{ }_{\text {left }}$ and $c_{\text {left }}$. Similarly, we refer to these measures for the right cue trials as $d^{\prime}{ }_{\text {right }}$ and $c_{\text {right }}$. Since we were not interested in general performance differences between leftward and rightward motion, we then averaged across these motion types to obtain $d^{\prime}$ predictive, which reflects the discriminability of the motion stimuli in the context of a predictive cue. On the other hand, $c_{\text {left }}$ and $c_{\text {right }}$ are the biases for answering "leftward" when a leftward or rightward cue was presented, respectively. Thus, we expected $c_{\text {left }}$ to be negative (corresponding to answering leftward $>50 \%$ of the time), and $c_{\text {right }}$ to be positive (corresponding to answering leftward $<50 \%$ of the time). Criterion shift was defined as $c_{\text {right }}-c_{\text {left }}$, i.e., the difference of how much the cues were able to move subjects' criterion for picking one response option over the other. The bigger this shift was, the more the subject adjusted their behavior based on the predictive cue. Reaction times and error rates were subjected to a $3 \times 3$ repeated-measures ANOVA with the factors cue validity (valid, invalid, neutral) and motion coherence (low, medium, high). $d^{\prime}$ and $c$ were subjected to a $2 \times 3$ repeated-measures ANOVA with the factors cue type (predictive, neutral) and motion coherence (low, medium, high). To further qualify the direction of the criterion shift for left- and right-cued trials, we also quantified the presence and direction of the criterion shift for each cue type separately, using post hoc $t$ tests. For each comparison, we used a significance level of $p<0.05$. Although it could be argued that the fact that we perform several statistical tests may warrant a correction for multiple comparisons, we elected to report individual significance levels, as we are not particularly interested in the null hypothesis that none of the effects would be significant, but rather treat each test as an independent question.

MEG measurements. Ongoing brain activity was recorded using a whole-head MEG with 275 axial gradiometers (CTF Systems). Data were collected at $12 \mathrm{kHz}$, and down-sampled to $1200 \mathrm{~Hz}$. To prevent aliasing, an eighth-order elliptic infinite impulse response filter was used with a cutoff at one-fourth of the sampling frequency, a $0.1 \mathrm{~dB}$ pass band ripple, and $120 \mathrm{~dB}$ attenuation at the Nyquist frequency as an anti-aliasing filter. Head localization was monitored continuously during the experiment
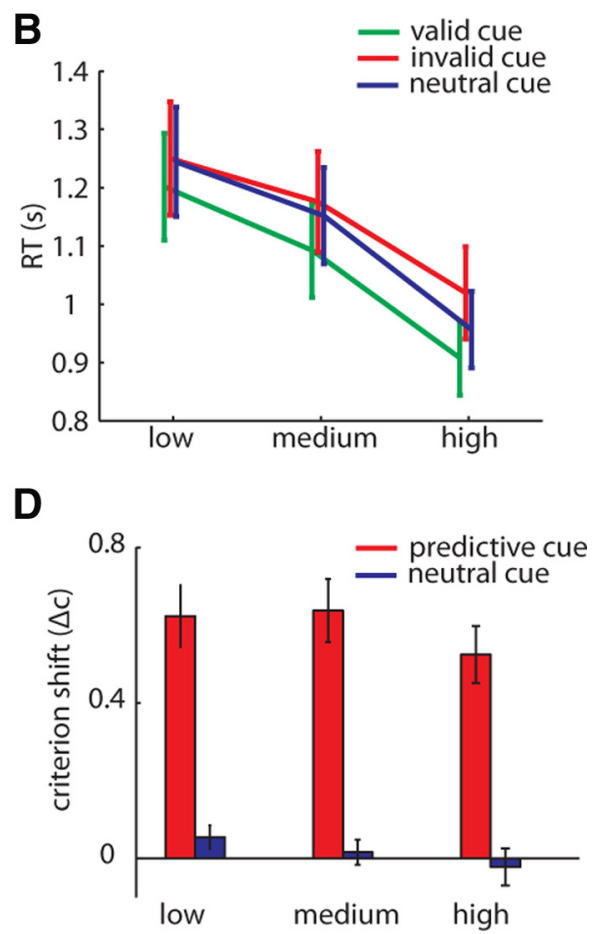

Figure 2. Behavioral results. $\boldsymbol{A}, \boldsymbol{B}$, Percentage correct $(\boldsymbol{A})$ and reaction times $(\boldsymbol{B})$ for trials that were preceded by a valid cue (green), invalid cue (red), or neutral cue (blue), as a function of motion coherence of the stimuli (low, medium, high). C, D, $d^{\prime}(\mathbf{C})$ and criterion shift $(\boldsymbol{D})$ for trials that were preceded by a predictive cue (red) or a neutral cue (blue), as a function of motion medium, high).

using coils that were placed at the cardinal points of the head (nasion, and left and right ear canal). The magnetic fields produced by these coils were used to measure the position of the subject's head with respect to the MEG sensor array. In addition to the MEG, the electrooculogram (EOG) was recorded from the supraorbital and infraorbital ridges of the left eye for the subsequent artifact rejection. Also, the electromyogram (EMG) and electrocardiogram (ECG) were recorded using 10-mm-diameter $\mathrm{Ag}-\mathrm{AgCl}$ surface electrodes. EMG electrodes were placed on the left and right forearm, in a belly-tendon arrangement, following standard skin preparation.

MEG data analysis. All MEG data analysis was performed using the FieldTrip toolbox developed at the Donders Institute for Brain, Cognition, and Behavior (Oostenveld et al., 2011) using Matlab 7 (MathWorks). Data were checked for artifacts using a semiautomatic routine that helped detecting and rejecting eye blinks, muscle artifacts, and jumps in the MEG signal caused by the SQUID electronics, using a twostep procedure. First, an infomax ICA algorithm (Delorme and Makeig, 2004) was used to decompose the signal into maximally independent sources, and components that were related to heartbeat and eyeblink artifacts were removed from the data. These components were identified pseudo-automatically, on the basis of their temporal correlation with the ECG and EOG signals, as well as their stereotypical topography. Subsequently, all data epochs of interest (between $2 \mathrm{~s}$ before stimulus onset until $500 \mathrm{~ms}$ after response onset) were visually inspected, and those contaminated by artifacts were removed. This inspection procedure was blind to condition and was based on the variance of the signal for each trial. On average, $2.4 \pm 1.8 \%$ (mean $\pm \mathrm{SD}$ ) of all trials were removed by this procedure.

An estimate of the planar gradient was calculated (Bastiaansen and Knösche, 2000). The horizontal and vertical components of the planar gradients were calculated for each sensor using the signals from the neighboring sensors, thus approximating the signal measured by MEG systems with planar gradiometers. The planar field gradient simplifies the interpretation of the sensor-level data because the maximal signal typically is located above the source (Hamalainen et al., 1993). For each sensor and each single trial, we calculated time-frequency representa- 
A

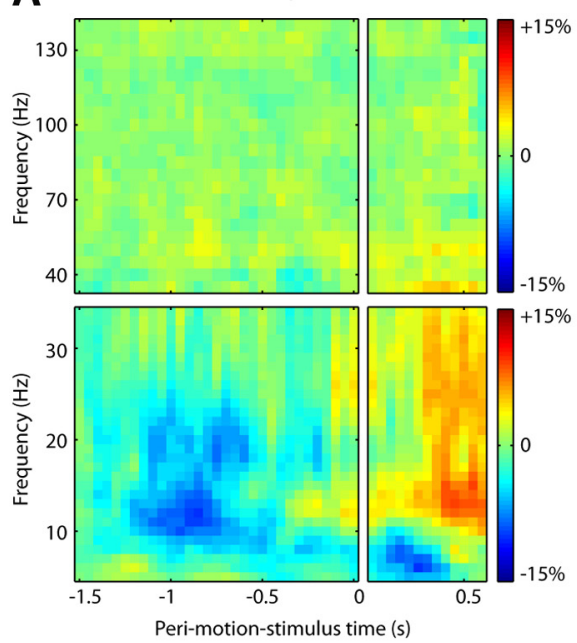

D

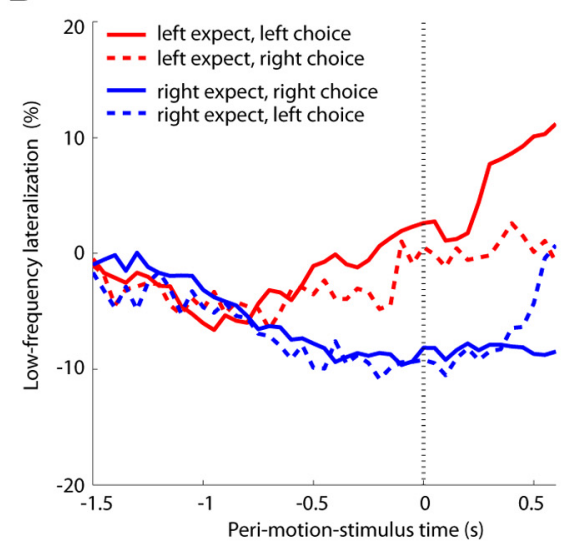

B

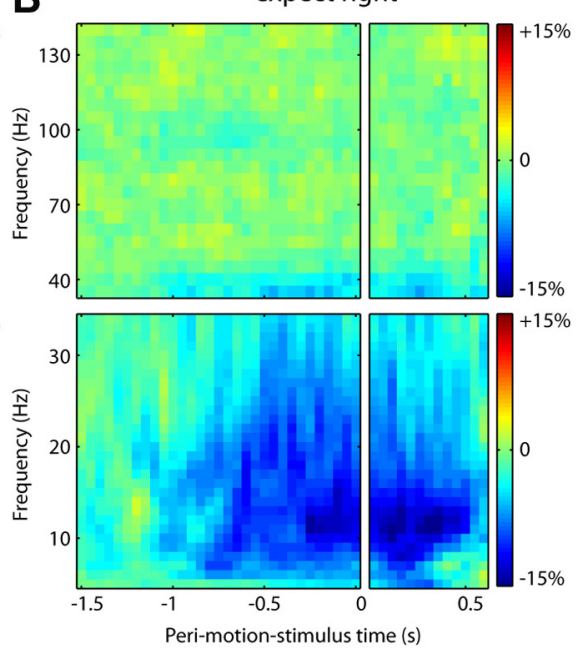

C expect left>right
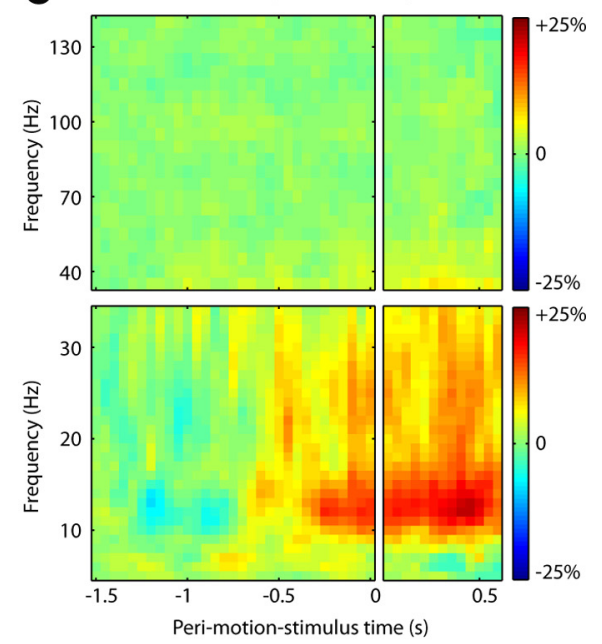

E
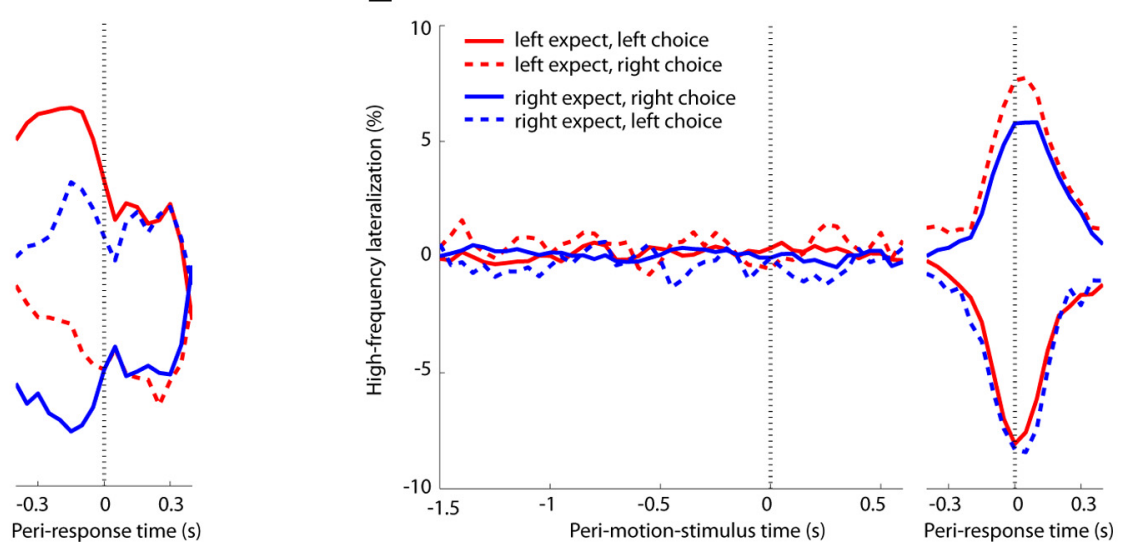

Figure 3. Expectation affects pre-motion stimulus motor-related activity. TFR of motor lateralization (decision signal, quantified as the difference between activity over the left and right sensors overlying the motor cortex; Fig. 1C) as a function of stimulus expectation. $\boldsymbol{A}-\boldsymbol{C}$, When comparing the decision signal for trials where subjects expect leftward motion $(\boldsymbol{A})$ with trials when they expect rightward motion $(\boldsymbol{B})$, there is a large difference in power in low frequencies $(\boldsymbol{C})$, as early as $600 \mathrm{~ms}$ before the onset of the motion stimulus. $\boldsymbol{D}$, Low-frequency $(8-30 \mathrm{~Hz})$ oscillatory activity as a function of expectation and decision. Expectation of leftward motion led to a positive prestimulus bias in the decision signal (red lines), expectation of rightward motion led to a prestimulus negative bias (blue lines). When subjects' expectation was different from the eventual decision (dotted red and blue lines), there was a gradual reversal of decision-related activity after motion stimulus onset. $\boldsymbol{E}$, Same as $\boldsymbol{D}$, but for high-frequency $(60-90 \mathrm{~Hz})$ oscillatory activity. High-frequency activity dissociated the eventual choice of the subjects, but we did not find any evidence of prestimulus modulation by expectation.

tions (TFRs) of the MEG power using a Fourier transform approach applied to short sliding time windows (Mitra and Pesaran, 1999). One (low frequencies) or three (high frequencies) "tapers" were multiplied with the data for each time window, and the result was submitted to a Fourier transform. Power estimates were averaged across tapers. The power values were calculated for the horizontal and vertical components of the planar gradient and then summed. We used the median of the planar gradient power estimates for all trials within a condition as our measure of MEG power for a given location in sensor space. We used the median because as a summary statistic it is less sensitive to extreme values (outliers) than the mean, i.e., it is a more robust statistic. For the lower $(5-35 \mathrm{~Hz})$ frequency range, we used a single Hanning taper and applied an adaptive time window $(T)$ of four cycles for each frequency $(\Delta T=$ $4 / f)$ which resulted in an adaptive smoothing of $\Delta f=1 / \Delta T$. For the higher $(35-140 \mathrm{~Hz})$ frequency range, we used a fixed taper length of 200 ms with a $\Delta f=20 \mathrm{~Hz}$ frequency smoothing (Percival and Walden, 1993). The percentage of change in power was calculated with respect to the frequency-specific power during a baseline window, which was centered $\sim 500-300 \mathrm{~ms}$ before the presentation of the cue. The window length of the baseline interval was equal to the window length of each estimated time point of interest (i.e., four cycles for the low-frequency range, and fixed at $200 \mathrm{~ms}$ for the higher frequency range).
TFRs of motor lateralization of power (sensors overlying left vs right motor cortex, see below) or occipital power (sensors overlying visual cortex, see below) were statistically compared between different conditions using nonparametric cluster-based permutation $t$ tests (Maris and Oostenveld, 2007). This type of test controls the type I error rate in the context of multiple comparisons by identifying clusters of significant differences over space, time, and/or frequency instead of performing a separate test on each sensor, sample, and frequency pair. For the quantification of motor-related lateralization (Fig. $1 \mathrm{~A}$ ), we identified the 30 channels (15 left and 15 right channels) that showed strongest lateralization in low-frequency $(8-30 \mathrm{~Hz})$ power during the 500 ms preceding the choice, when contrasting left with right motor cortex, in line with earlier work (Donner et al., 2007, , 2009). Quantification on the basis of highfrequency $(60-90 \mathrm{~Hz})$ power resulted in a highly overlapping topography, with power values of opposite sign (Fig. $1 A$, compare top, bottom; spatial correlation $r=-0.79, p<0.001)$. For the quantification of occipital activity modulations (Fig. $1 B$ ), we identified the 30 channels that showed the overall strongest increase in high-frequency $(60-140 \mathrm{~Hz})$ oscillatory power during the stimulus period (200-500 ms after stimulus onset), in line with an earlier study (Siegel et al., 2007).

For both analyses, we averaged over the spatial (channel) dimension, based on independent localization of the relevant channels. As such, our 
A
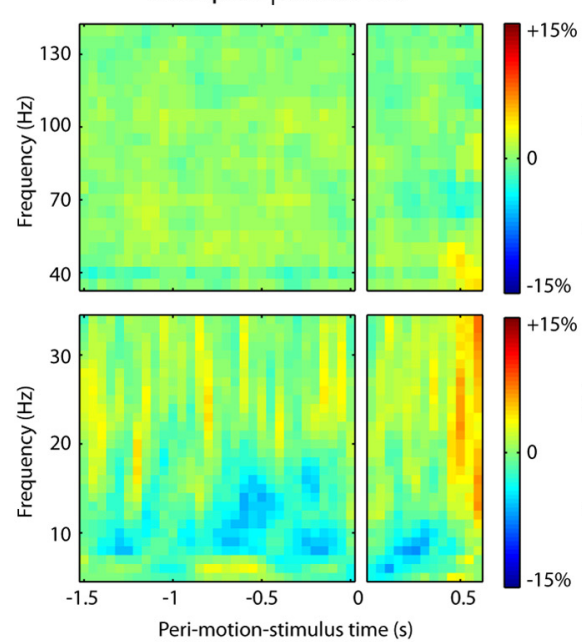

D

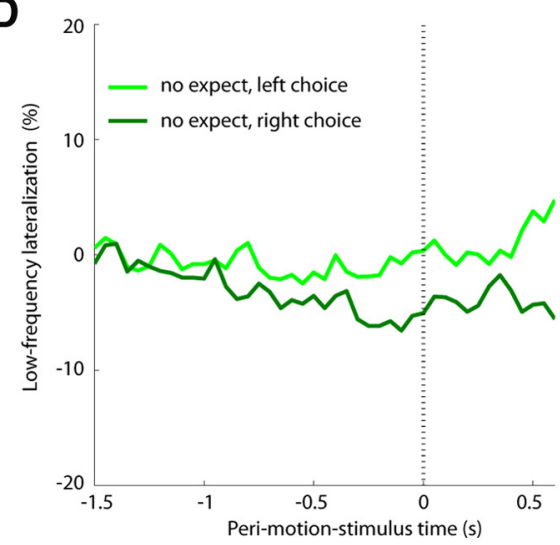

B

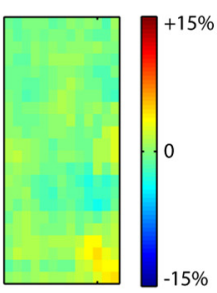

$15 \%$

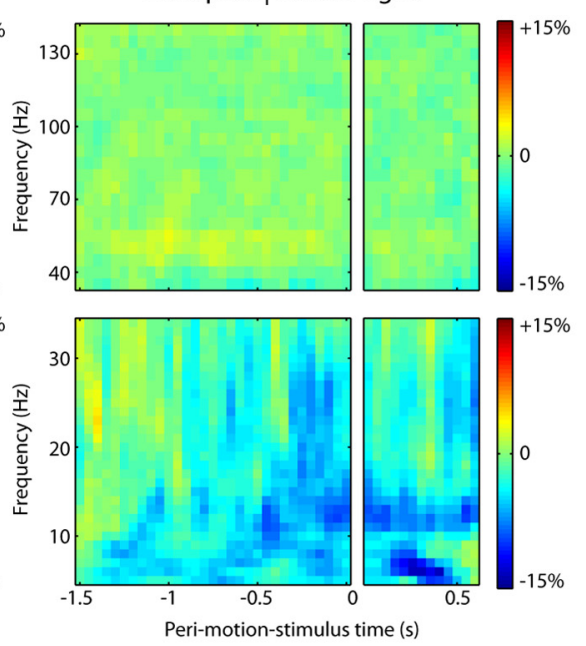

C no expect $\mid$ decide left>right

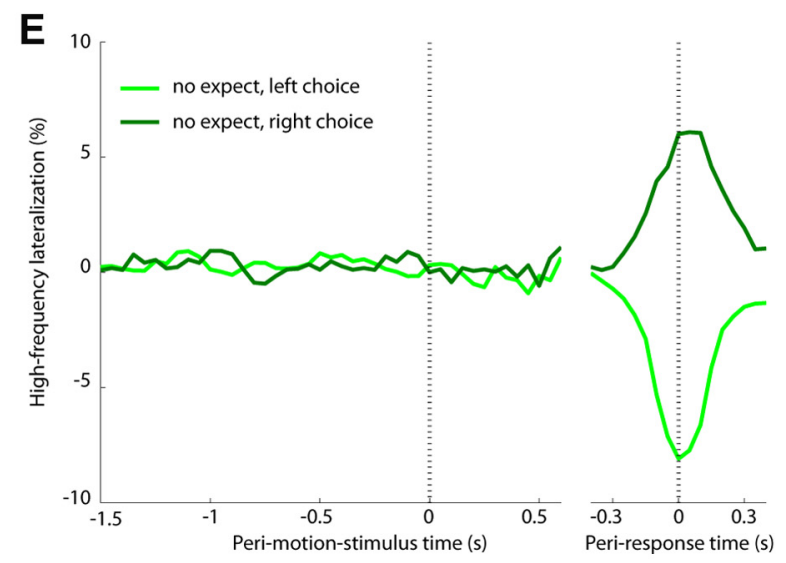

Figure 4. Spontaneous fluctuations in pre-motion stimulus motor-related activity on neutrally cued trials. TFR of motor lateralization during trials with no explicit expectation. $\boldsymbol{A}-\boldsymbol{C}$, When comparing the decision signal for neutrally cued trials where subjects decided leftward motion $(\boldsymbol{A})$ with trials when they decided rightward motion $(\boldsymbol{B})$, there is a significant pre-motion stimulus lateralization ( $\boldsymbol{C}$, which became significant $250 \mathrm{~ms}$ before the onset of the motion stimulus. $\boldsymbol{D}$, Low-frequency ( $8-30 \mathrm{~Hz}$ ) oscillatory activity for neutral trials. Pre-motion stimulus activity (from 250 ms preceding the stimulus) was predictive of eventual choice. $\boldsymbol{E}$, Same as $\boldsymbol{D}$, but for high-frequency $(60-90 \mathrm{~Hz})$ oscillatory activity.

statistical analysis considered two-dimensional (spectro-temporal) clusters. All cluster-level statistics, defined as the sum of $t$ values within each cluster, were evaluated under the permutation distribution of the maximum (minimum) cluster-level statistic. This permutation distribution was approximated by drawing 1000 random permutations of the observed data. The obtained $p$ values represent the probability under the null hypothesis (no difference between the conditions) of observing a maximum (minimum) cluster-level statistic that is larger (smaller) than the observed cluster-level statistics. We used this method to assess whether there were significant spectro-temporal (TFR) clusters of differential activity.

To assess whether there was a relationship between the behaviorally observed bias induced by the expectation cue and the neurally observed bias, we performed several correlation analyses. For the motor lateralization, we computed the motor lateralization difference between "expect left" and "expect right" trials for each individual subject. A larger value corresponds to a bigger difference in lateralization between expect left and expect right trials. Similarly, for the occipital alpha power difference, we calculated the magnitude of the power difference between "expectation" and "no-expectation" trials (the difference between cued and neutral conditions). These measures were correlated with the behaviorally observed criterion shift, calculated by subtracting the criterion used for expect left from the criterion used for expect right trials (larger values therefore correspond to subjects that were more strongly biased by the cues).

Finally, we assessed whether motor lateralization showed a modulation by motion coherence after stimulus onset (Donner et al., 2009). To compare the accumulation rates for different levels of coherence with maximal sensitivity, we averaged across trials with leftward and rightward motion, taking into account the sign of the motor lateralization. We then calculated and compared the slopes of motor lateralization after stimulus onset (in the $200-500$ ms poststimulus), as well as before response onset (in the $500 \mathrm{~ms}$ window preceding the response), using a linear regression approach.

\section{Results}

\section{Behavioral results}

As expected, subjects were less error prone and faster during trials with larger motion coherence [error rate $(\mathrm{ER}): F_{(2,36)}=54.6, p<$ 0.001 ; reaction time $(\mathrm{RT}): F_{(2,36)}=18.2, p<0.001$; Figure $\left.2 A, B\right]$. Subjects were also less error prone and faster when the direction of the motion in the stimulus matched the prior expectation (ER: $F_{(2,36)}=47.3, p<0.001$; RT: $\left.F_{(2,36)}=10.3, p<0.001\right)$. The effects of cue validity on RT were slightly larger when motion coherence was high (motion $\times$ cue: $F_{(4,72)}=2.64, p=0.041$ ), while the effects of cue validity on ER were larger when motion coherence was low (motion $\times$ cue: $\left.F_{(4,72)}=22.5, p<0.001\right)$.

We next investigated the effect of the predictive cues on discrimination sensitivity and bias, as measured with the signal detection measures $d^{\prime}$ and $c$ We computed $d^{\prime}$ predictive using all the valid and invalid trials and $d^{\prime}$ neutral using all the neutral trials. The 
key point to note about the procedure is that to compute $d^{\prime}$ predictive we separated the predictive (valid and invalid) trials into two categories: one in which the cue indicated that the upcoming stimulus was likely to be leftward $\left(d^{\prime}{ }_{\text {left }}\right)$ and one in which the cue indicated a likely rightward stimulus $\left(d^{\prime}{ }_{\text {right }}\right)$. Then, $d^{\prime}$ predictive was simply the average of $d^{\prime}{ }_{\text {left }}$ and $d^{\prime}{ }_{\text {right }}$ (see Materials and Method for more details). Similarly, we computed the signal detection measure of bias $\mathrm{c}$ for predictive $\left(c_{\text {predictive }}=c_{\text {right }}-c_{\text {left }}\right)$ and neutral $\left(c_{\text {neutral }}\right)$ trials.

As expected, $d^{\prime}$ increased with larger motion coherence $\left(F_{(1,18)}=48.5, p<0.001\right.$; Fig. $\left.2 C\right)$. There was a an influence of the cues on $d^{\prime}$ that interacted with motion coherence, such that stimulus processing sensitivity (independent of the aid provided by the cue) became worse after the presentation of a predictive cue, particularly for the highest coherence level (cue $\times$ motion coherence interaction: $\left.F_{(2,36)}=3.31, p=0.048\right)$. Post hoc tests indicated that $d^{\prime}$ was indeed significantly lower for predictive compared with neutral cues for high motion coherence $(p=$ $0.035)$ but not for low $(p=0.17)$ or medium $(p=0.74)$ coherence. Finally, predictive cues led to a large criterion shift $\left(F_{(1,18)}=53.7, p<0.001\right.$; Fig. $\left.1 D\right)$, which did not interact with the amount of motion coherence $\left(F_{(2,36)}=2.02, p=0.15\right)$. Post hoc tests showed that criterion was negative when trials were leftward cued $\left(t_{(18)}=-7.61, p<0.001\right)$ and positive when trials were rightward cued $\left(t_{(18)}=7.57, p<0.001\right)$, while there was no significant deviation from a zero bias for neutrally cued trials $\left(t_{(18)}=0.59, p=0.56\right)$.

\section{Expectations bias choice-selective low-frequency motor activity}

Perceptual (leftward/rightward motion) decisions were, by design, coupled to button presses with the left/right hand. Therefore, we used the choice-selective lateralization of activity over motor cortex as a proxy for the dynamics of decision-related brain activity during the trial (Donner et al., 2009). Indeed, immediately preceding the decision $(-250$ to $0 \mathrm{~ms}$ before the button press), there was suppression of low-frequency power (8-30 $\mathrm{Hz})$, and enhancement of high-frequency power $(60-90 \mathrm{~Hz})$, over the motor areas contralateral to the upcoming movement (Fig. 1A). We operationalized the "motor decision signal" by taking the difference in oscillatory power between the 15 left and 15 right sensors that were showing maximal oscillatory power differences. We then assessed whether there were any activity differences in the interval preceding the stimulus, as a function of prior expectation. When comparing expectation of leftward (Fig. $3 A$ ) with rightward (Fig. $3 B$ ) motion, there was a significant difference in the decision signal in low frequencies (spanning both the alpha- and beta-band), which was significant from $600 \mathrm{~ms}$ preceding stimulus onset [significant temporospectral cluster with time range $(-0.6-0 \mathrm{~s})$ and frequency range $(7-34) \mathrm{Hz}$; $p_{\text {cluster }}=0.0015$; Fig. $3 C$ ]. Thus, perceptual expectations about the upcoming stimulus were manifest in a motor bias before the onset of the visual stimulus. To assess the spatial specificity of this phenomenon, we inspected the topographical distribution of the expectation-related effect (Fig. 1C). Indeed, the topography of the prestimulus motor bias is highly similar to the prechoice topography (Fig. $1 A$, bottom; spatial correlation: $r=0.84, p<$ $0.001)$. The modulation of motor cortical oscillations by expectation was confined to low-frequency oscillations: although the gamma-band activity $(60-90 \mathrm{~Hz})$ clearly dissociated leftward from rightward choice at the moment of the overt choice, we did not find any evidence for prestimulus or poststimulus modulation of gamma-band motor cortical activity as a function of per-
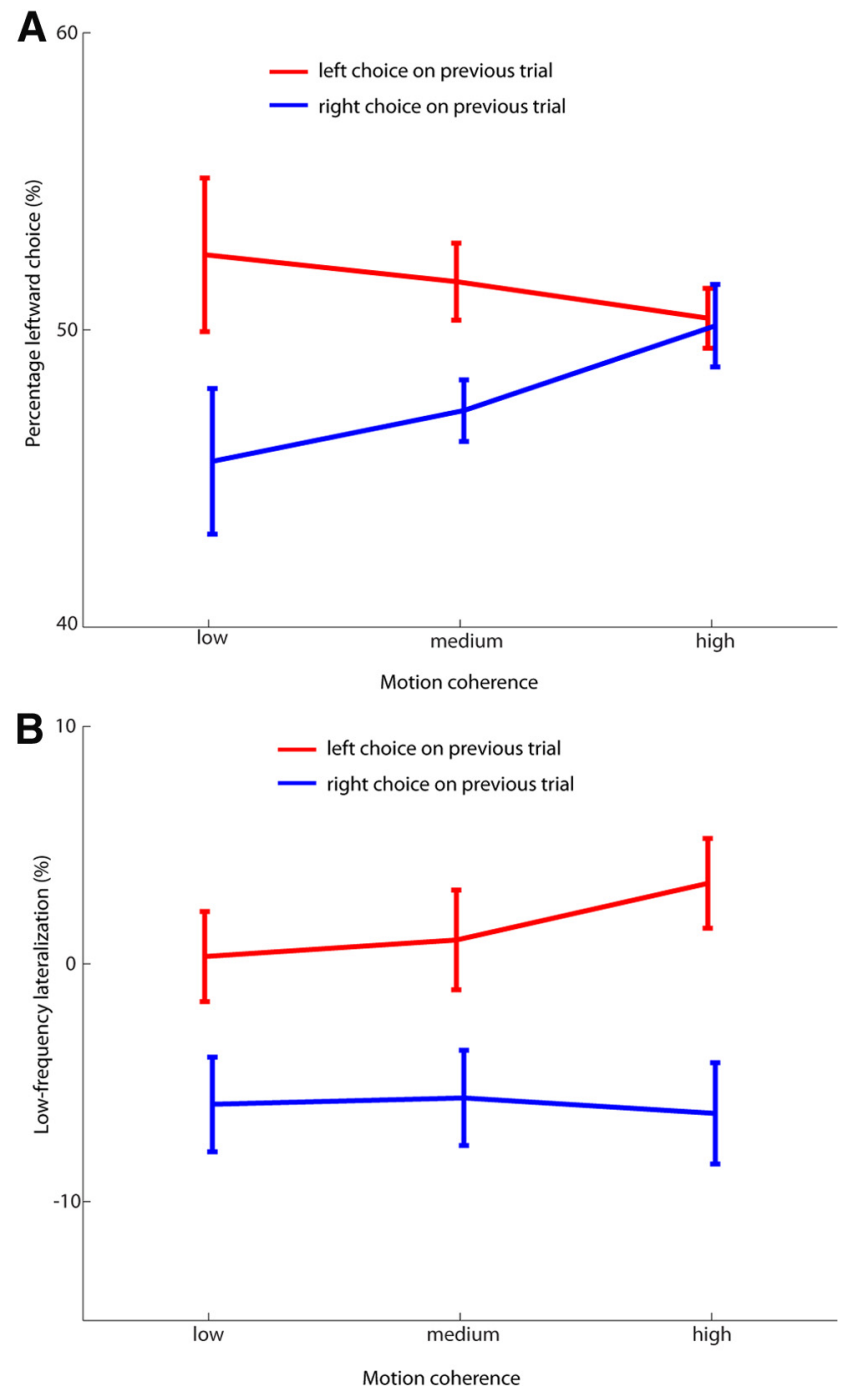

Figure 5. Sequential effects on neutrally cued trials. $A$, Behavioral bias. Percentage of leftward choices on neutrally cued trials as a function of participants' choice on the previous trial and motion coherence on the current trial. $\boldsymbol{B}$, Neural bias. Prestimulus motor lateralization on neutrally cued trials as a function of participants' choice on the previous trial (red and blue, left and right choice on previous choice respectively) and motion coherence on the current trial.

ceptual expectation ( $p>0.10$; Fig. $3 E$ ). The expectation-induced lateralization of low-frequency activity was dissociable from final choice (Fig. 3D): prestimulus lateralization was similar for trials where subjects did or did not change their decision on the basis of the sensory evidence $(p>0.10)$. In the latter case, there was a gradual poststimulus reversal of the decision signal (Fig. 3D, dotted lines). This decoupling is caused by the fact that the final decision is not fully governed by the prestimulus bias but also by the sensory stimulus itself, i.e., on a proportion of trials the subject decides in a direction that deviates from the cue.

\section{Spontaneous low-frequency motor activity and sequential effects}

On a subset of trials (33\%), subjects did not receive an informative cue about upcoming motion direction (neutral trials). Hence, on these trials they did not have any explicit expectation about upcoming motion direction. Therefore, these trials allowed us to probe whether spontaneous biases would also be visible in prestimulus activity modulations over the motor cortex. To investigate this issue, we conditioned all neutral trials on 
A

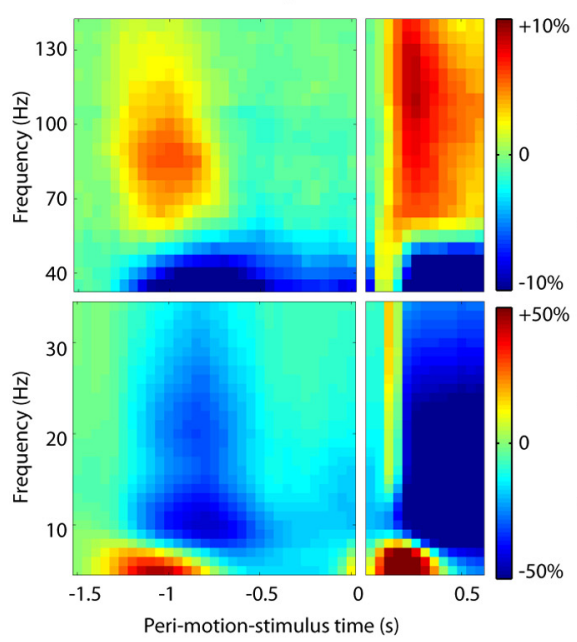

D

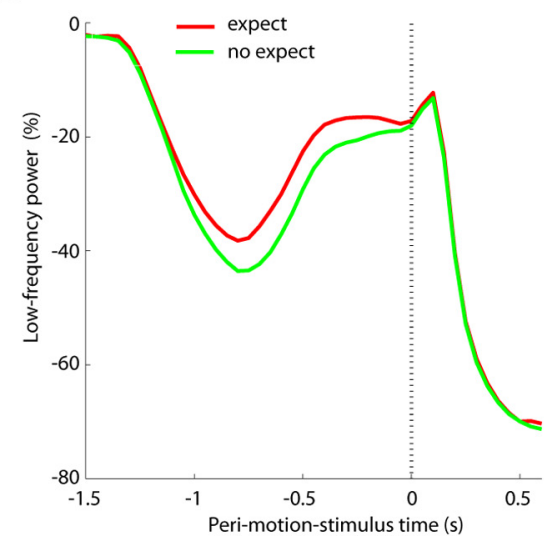

B

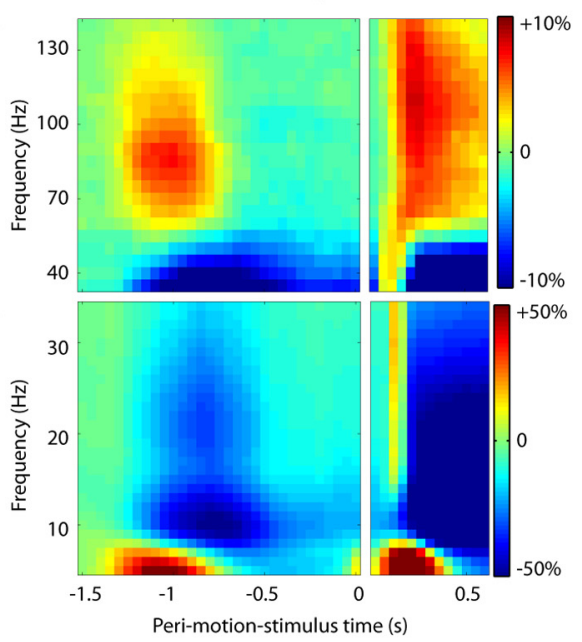

C

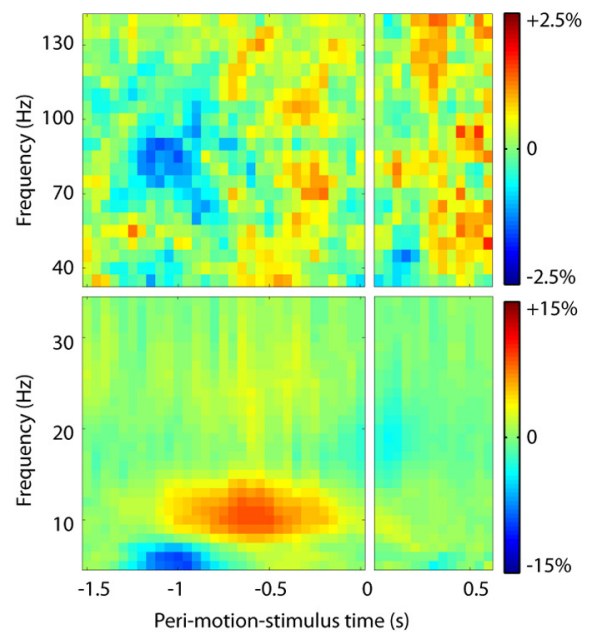

E
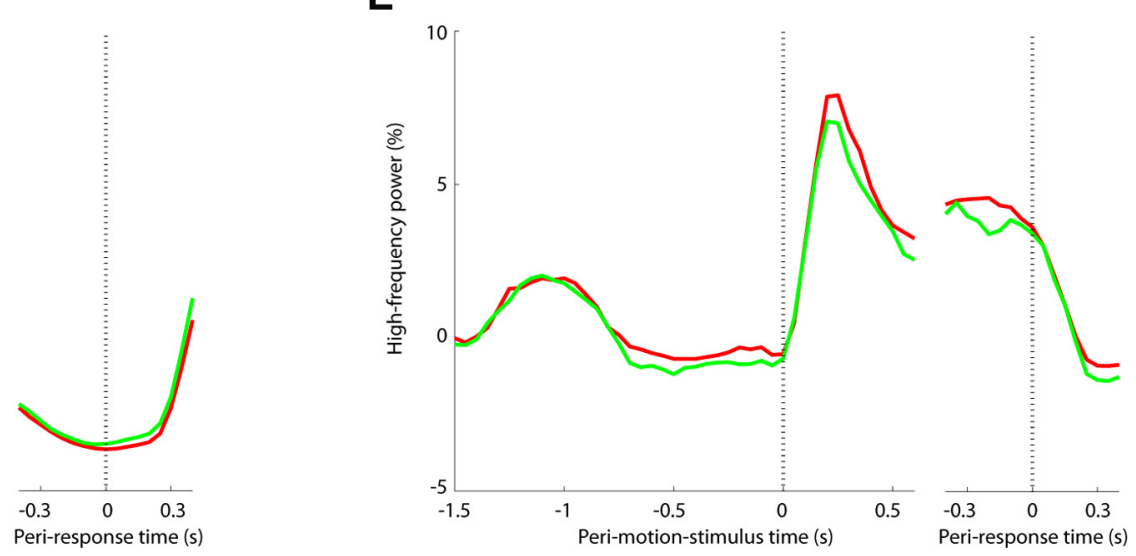

Figure 6. Expectation affects prestimulus visual activity. TFR of visual activity (Fig. 1D) as a function of stimulus expectation. $A-C$, When comparing the decision signal for trials where subjects had an expectation $(\boldsymbol{A})$ with trials when they had no expectation $(\boldsymbol{B})$, there is larger occipital power in low frequencies for trials in which subjects had an expectation $(\boldsymbol{C})$, throughout the prestimulus period. $\boldsymbol{D}$, Low-frequency $(7-15 \mathrm{~Hz})$ oscillatory activity as a function of expectation. $\boldsymbol{E}$, Same as $\boldsymbol{D}$, but for high-frequency $(60-140 \mathrm{~Hz})$ oscillatory activity.

the final choice of the subject (leftward vs rightward), and compared prestimulus activity between leftward (Fig. 4A) and rightward choices (Fig. 4B). If the choice is merely driven by the sensory evidence, we would not expect any prestimulus activity differences. In contrast, we observed a lateralization of prestimulus low-frequency power over motor cortex (Fig. 4C), which was significant from $250 \mathrm{~ms}$ preceding the stimulus onset and most strongly present in the beta-band [significant temporospectral cluster with time range $(-0.25$ to $-0.05 \mathrm{~s})$ and frequency range $(20-28 \mathrm{~Hz}), p_{\text {cluster }}=0.013$; Fig. $\left.4 D\right]$. This spontaneous bias was not statistically different for trials with high vs low motion coherence $(t=-1.35, p=0.20)$. We did not find evidence for a relation between spontaneous fluctuations in lateralization of motor gamma-band power and eventual choice $(p>0.10$; Fig. 4E).

To investigate the potential origin of these spontaneously emerging neural biases as well as their effect on behavior, we examined whether the neural biases were induced by previous choices (Leopold et al., 2002). Behavioral and computational studies of performance on two-choice decision making suggest a behavioral advantage for a particular choice when it has been selected on the immediately preceding trial (Gao et al., 2009). To examine the presence of such (first-order) sequential effects and their possible interaction with motion coherence (strongest se- quential effects are expected for low-coherence trials), we quantified the proportion of trials on which subjects chose for rightward motion, conditioned on the subject's choice on the preceding trial. We performed this analysis by focusing on the neutrally cued trials, and separately for the different levels of motion coherence on the current trial.

Indeed, there was a substantial effect of the previous trial on neural activity and behavior. Trials that were marked by a leftward choice on the preceding trial were biased toward a leftward choice on the current trial, and trials that were marked by a rightward choice on the preceding trial were biased toward a rightward choice on the current trial $(T=1.99, p=0.031$; Fig. $5 A)$. This bias tended to linearly decrease with increasing motion coherence $(T=1.68, p=0.055)$, with the largest bias for low coherence trials $(6.95 \%)$, an intermediate bias for intermediate coherence $(4.34 \%)$, and virtually no bias for high coherence trials $(0.25 \%)$. The bias was not present when conditioning on the previous cue, instead of the previous choice $(T=-0.65, p=$ 0.26 ), suggesting it reflects a leakage of the previous decision, rather than the previous cue. Next, we investigated prestimulus low-frequency lateralization in motor areas as a function of the preceding choice. Here, we also observed significant prestimulus lateralization on the current trial into the direction of the choice on the preceding trial $(T=2.56, p=0.010)$. This difference was 
present for all three motion coherence levels (all $p<0.05$ ) and was not modulated by coherence $(T=1.04, p=0.16)$ (Fig. $5 B)$. These results suggest that the decision on a previous trial indeed generates a decision bias on the current trial, which is visible in prestimulus motor lateralization, and the influence of which on behavior is dependent on the strength of the motion coherence of the impending stimulus.

Expectations bias prestimulus alpha power over visual cortex We did not expect to detect putative direction-selective biases in neural activity in visual cortex (presumably organized on a mesoscopic scale) (Liu and Newsome, 2006) with our macroscopic MEG measurements. But, as a proxy for "global" state changes in visual cortex due to expectation, we compared overall prestimulus visual activity, as defined in Figure $1 B$, on trials in which there was an expectation with those in which there was no expectation. Here, we observed larger alpha-band power over visual cortex during trials in which subjects had a prior expectation (leftward or rightward, Fig. $6 A$ ) than trials in which subjects did not have a prior expectation (neutral; Fig. 6B). This power difference was significant throughout the whole prestimulus period [significant temporospectral cluster with time range $(-1.05$ to $-0.1 \mathrm{~s})$ and frequency range $(7-15 \mathrm{~Hz})$; $p_{\text {cluster }}=0.010$; Fig. $6 \mathrm{C}$ ], longer than what could be explained by the length of the sliding window for this frequency range $(\sim 400 \mathrm{~ms})$. To assess the spatial specificity of this phenomenon, we inspected the topographical distribution of the visual expectation-related effect (Fig. 1D). The topography of the prestimulus increase in low-frequency power for trials in which subjects had a prior expectation provided a good match with the sensors that were marked by low-frequency suppression and high-frequency enhancement of oscillatory activity during the stimulus period (Fig. $1 B$; spatial correlation analysis: $r=0.58$, $p<0.001)$. This appears to rule out the possibility that the observed prestimulus effects of expectation over occipital sensors are driven by volume conduction of motor-related effects.

There was a small physical difference between the cue types: neutral cues were composed of more characters than predictive cues ( 7 vs 4.5 characters, see Materials and Methods). Accordingly, there was larger gamma-band power at the time of the cue presentation for neutral cues [significant temporospectral cluster with time range $(-1.2$ to $-0.95 \mathrm{~s})$ and frequency range (75-90 $\left.\mathrm{Hz}) ; p_{\text {cluster }}=0.047\right)$. This difference was however transient in nature, and markedly earlier than the low-frequency effects described above. Finally, there were no differences in low- or highfrequency power (Fig. $6 D, E$ ) after stimulus onset with either cue presence or validity $(p>0.10)$.

\section{Brain-behavior correlations}

Finally, we examined whether individual variability in perceptual/decision bias induced by the expectation cue correlated with prestimulus activity biasing of the choice-selective motor activity, as well as the occipital alpha increase. Indeed, there was a significant correlation between prestimulus motor lateralization and criterion shift $(r=0.54, p=0.009$; Fig. $7 A)$, as well as a significant correlation between prestimulus visual alpha increase and criterion shift ( $r=0.45, p=0.026$; Fig. $7 B)$. Indeed, the prestimulus motor lateralization and visual alpha increase were themselves significantly correlated $(r=0.40, p=0.047)$, possibly due to the fact that they were comodulated by the expectation cue. Thus, these two effects, which are manifest at the sensory and motor peripheries of the decision process, appear both related to the behaviorally induced biasing of choice.
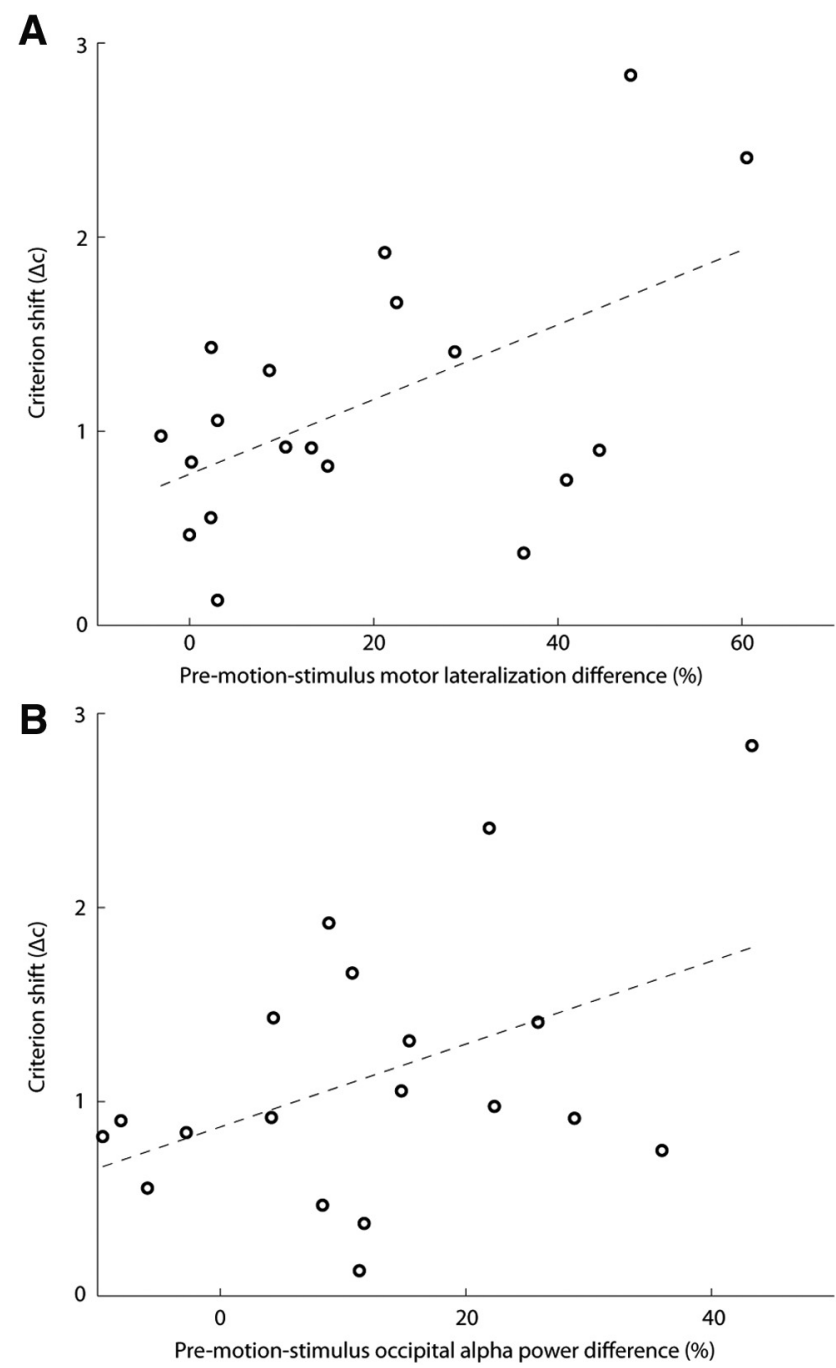

Figure 7. Correlation between behavioral and neural markers of expectation. $A$, Interindividual differences in prestimulus motor lateralization between trials with leftward vs rightward expectation (Fig. 3) correlated with the behaviorally observed criterion shift (Fig. 1D) as a result of the expectation cue. $\boldsymbol{B}$, Interindividual differences in prestimulus occipital low-frequency power between trials with versus without stimulus expectation (Fig. 4) also correlated with the behaviorally observed criterion shift (Fig. 1D) as a result of the expectation cue.

\section{Poststimulus motor lateralization}

We assessed whether motor lateralization showed a modulation by the strength of the sensory evidence after stimulus onset, as has been observed during a motion detection task (Donner et al., 2009). For this, we quantified the slope of motor lateralization in both the poststimulus and preresponse time interval. We expected a steeper poststimulus and preresponse slope with increasing motion coherence (Fig. 8A). In the interval after stimulus onset, the slopes of choice-selective motor activity were modest and not significantly different from each other $(F=0.20$, $p>0.10)$. In the preresponse period however, there was a significant difference in slope between the three motion coherence levels $(F=7.94, p=0.0014)$. Post hoc tests revealed that there was a significantly larger slope of choice-selective motor activity for the high than low coherence motion stimuli (Fig. $8 B$; slope $_{\text {high }}=$ 0.034 , slope $\left._{\text {low }}=0.021 ; \mathrm{T}=3.16, p=0.0027\right)$, with the medium coherence motion falling in between low and high coherence motion $\left(\right.$ slope $_{\text {medium }}=0.031$ ). 


\section{Discussion}

In this study, we examined how prior expectation influences perceptual decision making. Using MEG, we tracked choice-selective activity in the motor system while the decision process was unfolding. We reasoned that motor cortical activity may provide a window into the decision process since the different perceptual choices were coupled to different effectors (Coles et al., 1985; Tosoni et al., 2008; Donner et al., 2009; Song and Nakayama, 2009; Gould et al., 2012). We observed that prior expectation resulted in a strong prestimulus lateralization of motor activity in low frequencies (alpha and beta bands). Spontaneous biases in this lateralization, occurring in the absence of any explicitly induced expectation, were also related to upcoming perceptual choice, and were, at least in part, driven by the choice from the previous trial. During the stimulus period, these oscillations also tracked the fidelity of the perceptual signal. Expectations also led to increased lowfrequency oscillations in visual cortex before the onset of the stimulus. Finally, the extent to which expectation changed prestimulus motor lateralization and low-frequency oscillations in visual cortex within a given subject partly predicted the subjects' behavioral criterion shift induced by the expectation.
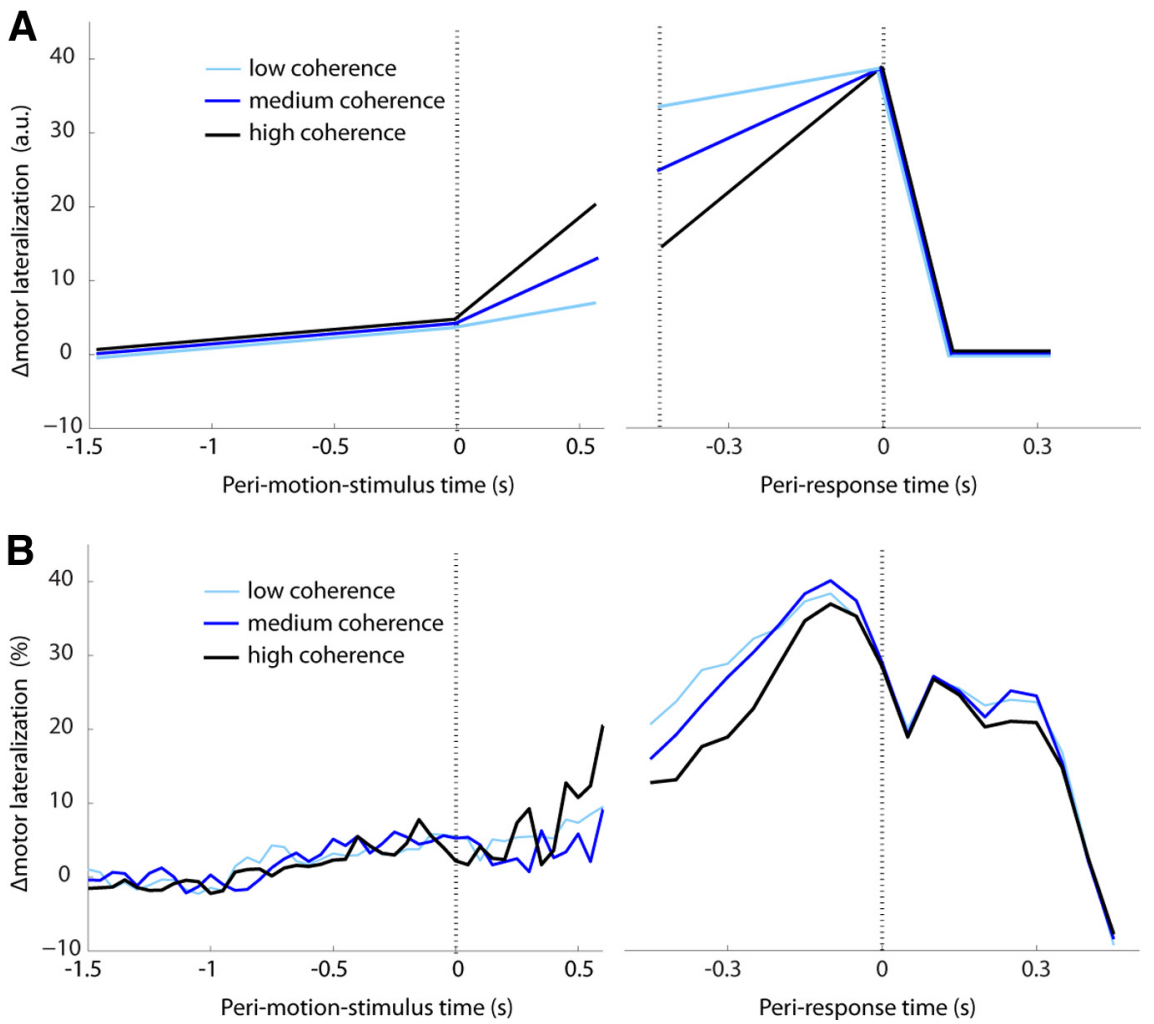

Figure 8. Poststimulus motor lateralization reflects integration of perceptual evidence. $A$, Expected pattern of results. Motor lateralization is expected to increase during the motion stimulus period, with the amount of increase proportional to the strength of the stimulus (motion coherence). $\boldsymbol{B}$, Observed pattern of results. Motor lateralization increased during the motion stimulus period. Although there was no significant difference in post-motion stimulus motor lateralization as a function of motion coherence, there was a significant difference in preresponse motor lateralization, in line with the expected pattern of results.

\section{Biasing mechanisms of perceptual choice}

Prior probability can change perceptual decision making in several ways, and at different stages of the decision process. Computational models of decision making have stipulated that prior probability, which has been manipulated by changing, for example, the relative proportions of two stimulus types, may lead to changes in the starting point, the gain of accumulation, or both. The presence of both types of effects have been inferred from reaction time and error rate distribution in human observers performing motion discrimination tasks (Ratcliff and McKoon, 2008), but the evidence conferred by these models is necessarily indirect. Neuroimaging studies using fMRI have asked a similar question: Which brain regions are affected by manipulating prior expectation during perceptual decision making: sensory areas collecting the evidence or decision-related areas accumulating the evidence? These studies have found evidence for biasing of activity of both the sensory areas encoding the evidence (Shulman et al., 1999; Preuschhof et al., 2010; Todorovic et al., 2011; Kok et al., 2012a,b) and associative areas related to action planning (Domenech and Dreher, 2010; Forstmann et al., 2010; Hanks et al., 2011), as well as the connectivity between sensory and associative cortical areas (Rahnev et al., 2011).

However, previous studies did not dynamically track the influence of prior probability on choice-predictive signals in the human brain. Further, no previous study directly compared such choice-selective cortical biases before stimulus onset, depending on whether they emerged spontaneously or were externally cued. Finally, no study has so far directly linked expectation effects in visual cortex to choice-selective biases in the motor cortex during perceptual choice tasks. The current study therefore provides a critical ex- tension of previous studies by noninvasively quantifying sensory and choice-selective activity using MEG and measuring its perturbation by prior probability. Overall, our results provide evidence for the notion that prior probability modifies the starting point of the motor decision variable, as demonstrated by the prestimulus lateralization of low-frequency motor cortical activity. Previous studies have shown that low-frequency oscillations, particularly in the beta band, are attenuated before and during voluntary movements (Jenkinson and Brown, 2011; Joundi et al., 2012). Therefore, our results are in line with earlier studies that observed increased excitability (Bestmann et al., 2008) and activity (Scheibe et al., 2010) in the motor system with increasing decision certainty. They are also congruent with neurophysiological data in monkeys showing that fluctuations in prestimulus firing rates in the lateral intraparietal cortex predict upcoming perceptual choice, especially when the evidence conferred by the stimulus is weak (Shadlen and Newsome, 2001), and are selectively increased by prior expectations (Rao et al., 2012).

Our results also suggest changes in visual cortex contingent on the presence of a prior expectation (Fig. 6), as suggested previously (Schlack and Albright, 2007). However, our results lack the spatial resolution to make strong claims about the underlying mechanism of this biasing process. We speculate that the expectation-related effect over occipital cortex could reflect a state of overall reduced excitability (Jensen and Mazaheri, 2010), akin to an attentional modulation that is driven by the expectation cue (Summerfield and Egner, 2009). More specifically, since retinotopically specific alpha-band power reductions are indicative of increased visuospatial attention (Worden et al., 2000), we believe that the larger low-frequency power reduction for no- 
expectation trials could reflect the fact that a larger population of neurons (i.e., direction-selective cells for both motion directions) is released from inhibition (Jensen and Mazaheri, 2010) in these trials than for the expectation trials, where only the relevant (expected) subpopulation of neurons is released from inhibition. A limitation of the current study is that our expectation cues were perceptually not completely matched (i.e., they were slightly larger for neutral than non-neutral cues), which could potentially constitute an alternative explanation for the effect of expectation observed in the visual cortex. Although we cannot exclude this possibility, the fact that the magnitude of the neural effect of expectation correlated with the magnitude of the behaviorally observed criterion shift effect, and occurred in a period that was more than $\sim 500-600 \mathrm{~ms}$ after the offset of the cue presentation, suggest, however, that the neural effect in visual cortex is related to the cognitive/behavioral consequences of the cue, rather than its physical difference.

\section{Relationship between motor bias and perceptual choice}

In our experiment, we coupled perceptual choice to particular effector choices, allowing us to read out the choice-related activity from the motor cortex. This, however, begs the question whether the activity modulations that we observed may constitute a response bias that is independent from any perceptual biases that are potentially induced by the expectation cues. Although the motor lateralization is undoubtedly a reflection of motor preparation, there are several arguments for why this motor preparation signal may, at least under the current experimental conditions, provide a window into the decision process unfolding in the brain. First, previous studies have shown a tight relationship between the choice-selective motor preparatory activity and the temporal integral of sensory evidence, both in monkeys for review, see (Gold and Shadlen, 2007) and in humans (Donner et al., 2009; Siegel et al., 2011; Gould et al., 2012). As such, there is converging evidence that perceptual and cognitive processes "leak into" the motor system (Song and Nakayama, 2009). Indeed, also in our study we observed an increase in motor preparatory activity during the stimulus encoding period that is suggestive of an integration process of perceptual evidence (Fig. $8)$. Second, there were concomitant changes in visual cortex induced by the expectation cues, in the form of stronger alpha-band power when subjects had an expectation about upcoming motion direction compared with no expectation. Notably, the individual strength of the behavioral choice bias induced by the expectation correlated with the individual strength of both the alpha-power increase in occipital cortex and choice-selective activity in motor cortex. This suggests that these neuronal signatures of expectation evident at the sensory and motor peripheries of the decision process may in fact be produced by the same biasing mechanism.

Interestingly, we observed that spontaneous fluctuations in motor lateralization, in the absence of any explicit cueing about the probable stimulus/response, also partly predicted upcoming choice. Thus, even in the absence of explicit cues, the state of motor cortex has repercussions for perceptual choice. An analogous pattern of results has been previously found in single-unit activity in the monkey lateral and ventral intraparietal areas (Shadlen and Newsome, 2001; Williams et al., 2003). In view of the tight and bidirectional links between cortical sensory and motor systems, it is possible that these spontaneous motor fluctuations may be a downstream reflection of spontaneous fluctuations in sensory cortices (Leopold et al., 2002; Wilke et al., 2006; Hesselmann et al., 2008; Berkes et al., 2011), or vice versa. We also observed a possible source for the generation of these spontane- ous fluctuations. Namely, decisions on the immediately previous trial resulted in a significant prestimulus lateralization on the current trial into the direction of the choice on the preceding trial, in line with computational models of two-choice behavior (Gao et al., 2009). Interestingly, this neural bias interacted with motion coherence, with diminishing influence of the prestimulus bias on behavior with increasing poststimulus strength (Fig. 5).

Our results do not imply that the motor cortex itself accumulates the sensory evidence during the decision process (Gold and Shadlen, 2003; Donner et al., 2009; Siegel et al., 2011). Indeed, it is likely that observers can accumulate sensory evidence in the absence of a corresponding motor plan. In line with this, we (Rahnev et al., 2011) and others (Heekeren et al., 2004; Ho et al., 2009) have previously isolated neural activity that is suggestive of an evidence accumulation signal in the dorsolateral prefrontal cortex-outside the motor system-when perceptual evidence was not directly mapped to particular responses. Integrating these former studies with the current study, we speculate that prior expectation may bias activity in associative cortical areas, such as the posterior parietal and dorsolateral prefrontal cortex, which broadcast the decision variable to motor cortex when the decisions can be mapped onto a particular motor effector.

\section{Conclusions}

We found that prior expectation affects perceptual choice behavior by altering the state of both visual and motor cortices. In particular, perceptual expectations bias the lateralization of prestimulus low-frequency activity in motor cortex and the amount of low-frequency activity in visual cortex. Moreover, spontaneous fluctuations in motor cortex lateralization could partly predict upcoming perceptual choice. Together, these results show how perceptual expectations leak into the motor system to facilitate flexible and adaptive decision making.

\section{References}

Bastiaansen MC, Knösche TR (2000) Tangential derivative mapping of axial MEG applied to event-related desynchronization research. Clin Neurophysiol 111:1300-1305. CrossRef Medline

Berkes P, Orbán G, Lengyel M, Fiser J (2011) Spontaneous cortical activity reveals hallmarks of an optimal internal model of the environment. Science 331:83-87. CrossRef Medline

Bestmann S, Harrison LM, Blankenburg F, Mars RB, Haggard P, Friston KJ, Rothwell JC (2008) Influence of uncertainty and surprise on human corticospinal excitability during preparation for action. Curr Biol 18:775780. CrossRef Medline

Bogacz R, Brown E, Moehlis J, Holmes P, Cohen JD (2006) The physics of optimal decision making: a formal analysis of models of performance in two-alternative forced-choice tasks. Psychol Rev 113:700-765. CrossRef Medline

Brainard DH (1997) The Psychophysics Toolbox. Spat Vis 10:433-436. CrossRef Medline

Chalk M, Seitz AR, Series P (2010) Rapidly learned stimulus expectations alter perception of motion. J Vis 10(8):2 1-18. CrossRef Medline

Churchland AK, Kiani R, Shadlen MN (2008) Decision-making with multiple alternatives. Nat Neurosci 11:693-702. CrossRef Medline

Coles MG, Gratton G, Bashore TR, Eriksen CW, Donchin E (1985) A psychophysiological investigation of the continuous flow model of human information processing. J Exp Psychol 11:529-553. CrossRef

Delorme A, Makeig S (2004) EEGLAB: an open source toolbox for analysis of single-trial EEG dynamics including independent component analysis. J Neurosci Methods 134:9-21. CrossRef Medline

Domenech P, Dreher JC (2010) Decision threshold modulation in the human brain. J Neurosci 30:14305-14317. CrossRef Medline

Donner TH, Siegel M, Oostenveld R, Fries P, Bauer M, Engel AK (2007) Population activity in the human dorsal pathway predicts the accuracy of visual motion detection. J Neurophysiol 98:345-359. CrossRef Medline

Donner TH, Siegel M, Fries P, Engel AK (2009) Buildup of choice- 
predictive activity in human motor cortex during perceptual decision making. Curr Biol 19:1581-1585. CrossRef Medline

Forstmann BU, Brown S, Dutilh G, Neumann J, Wagenmakers EJ (2010) The neural substrate of prior information in perceptual decision making: a model-based analysis. Front Hum Neurosci 4:40. CrossRef Medline

Gao J, Wong-Lin K, Holmes P, Simen P, Cohen JD (2009) Sequential effects in two-choice reaction time tasks: decomposition and synthesis of mechanisms. Neural Comput 21:2407-2436. CrossRef Medline

Gold JI, Shadlen MN (2003) The influence of behavioral context on the representation of a perceptual decision in developing oculomotor commands. J Neurosci 23:632-651. Medline

Gold JI, Shadlen MN (2007) The neural basis of decision making. Annu Rev Neurosci 30:535-574. CrossRef Medline

Gould IC, Nobre AC, Wyart V, Rushworth MF (2012) Effects of decision variables and intraparietal stimulation on sensorimotor oscillatory activity in the human brain. J Neurosci 32:13805-13818. CrossRef Medline

Green DM, Swets JA (1966) Signal detection theory and psychophysics. New York: Wiley.

Hamalainen MH, Hari R, Ilmoniemi RJ, Knuutila J, Lounasmaa J (1993) Magnetoencephalography. Theory, instrumentation and applications to the noninvasive study of brain function. Rev Mod Phys 65:413-497. CrossRef

Hanks TD, Mazurek ME, Kiani R, Hopp E, Shadlen MN (2011) Elapsed decision time affects the weighting of prior probability in a perceptual decision task. J Neurosci 31:6339-6352. CrossRef Medline

Heekeren HR, Marrett S, Bandettini PA, Ungerleider LG (2004) A general mechanism for perceptual decision-making in the human brain. Nature 431:859-862. CrossRef Medline

Heekeren HR, Marrett S, Ungerleider LG (2008) The neural systems that mediate human perceptual decision making. Nat Rev Neurosci 9:467479. CrossRef Medline

Hesselmann G, Kell CA, Kleinschmidt A (2008) Ongoing activity fluctuations in hMT + bias the perception of coherent visual motion. J Neurosci 28:14481-14485. CrossRef Medline

Ho TC, Brown S, Serences JT (2009) Domain general mechanisms of perceptual decision making in human cortex. J Neurosci 29:8675-8687. CrossRef Medline

Jenkinson N, Brown P (2011) New insights into the relationship between dopamine, beta oscillations and motor function. Trends Neurosci 34: 611-618. CrossRef Medline

Jensen O, Mazaheri A (2010) Shaping functional architecture by oscillatory alpha activity: gating by inhibition. Front Hum Neurosci 4:186. CrossRef Medline

Joundi RA, Jenkinson N, Brittain JS, Aziz TZ, Brown P (2012) Driving oscillatory activity in the human cortex enhances motor performance. Curr Biol 22:403-407. CrossRef Medline

Kok P, Jehee JF, de Lange FP (2012a) Less is more: expectation sharpens representations in the primary visual cortex. Neuron 75:265-270. CrossRef Medline

Kok P, Rahnev D, Jehee JF, Lau HC, de Lange FP (2012b) Attention reverses the effect of prediction in silencing sensory signals. Cereb Cortex 22: 2197-2206. CrossRef Medline

Leopold DA, Wilke M, Maier A, Logothetis NK (2002) Stable perception of visually ambiguous patterns. Nat Neurosci 5:605-609. CrossRef Medline

Liu J, Newsome WT (2006) Local field potential in cortical area MT: stimulus tuning and behavioral correlations. J Neurosci 26:7779-7790. CrossRef Medline

Macmillan NA, Creelman CD (2005) Detection theory: a user's guide. Mahwah, NJ: Erlbaum.

Maris E, Oostenveld R (2007) Nonparametric statistical testing of EEG- and MEG-data. J Neurosci Methods 164:177-190. CrossRef Medline

Mitra PP, Pesaran B (1999) Analysis of dynamic brain imaging data. Biophys J 76:691-708. CrossRef Medline

Oostenveld R, Fries P, Maris E, Schoffelen JM (2011) FieldTrip: open source software for advanced analysis of MEG, EEG, and invasive electrophysiological data. Comput Intell Neurosci 2011:156869. CrossRef Medline
Percival DB, Walden AT (1993) Spectral analysis for physical applications: multitaper and conventional univariate techniques. Cambridge: Cambridge UP.

Preuschhof C, Schubert T, Villringer A, Heekeren HR (2010) Prior Information biases stimulus representations during vibrotactile decision making. J Cogn Neurosci 22:875-887. CrossRef Medline

Rahnev D, Lau H, de Lange FP (2011) Prior expectation modulates the interaction between sensory and prefrontal regions in the human brain. J Neurosci 31:10741-10748. CrossRef Medline

Rao V, DeAngelis GC, Snyder LH (2012) Neural correlates of prior expectations of motion in the lateral intraparietal and middle temporal areas. J Neurosci 32:10063-10074. CrossRef Medline

Ratcliff R, McKoon G (2008) The diffusion decision model: theory and data for two-choice decision tasks. Neural Comput 20:873-922. CrossRef Medline

Schall JD (2001) Neural basis of deciding, choosing and acting. Nat Rev Neurosci 2:33-42. CrossRef Medline

Scheibe C, Ullsperger M, Sommer W, Heekeren HR (2010) Effects of parametrical and trial-to-trial variation in prior probability processing revealed by simultaneous electroencephalogram/functional magnetic resonance imaging. J Neurosci 30:16709-16717. CrossRef Medline

Schlack A, Albright TD (2007) Remembering visual motion: neural correlates of associative plasticity and motion recall in cortical area MT. Neuron 53:881-890. CrossRef Medline

Shadlen MN, Newsome WT (1996) Motion perception: seeing and deciding. Proc Natl Acad Sci U S A 93:628-633. CrossRef Medline

Shadlen MN, Newsome WT (2001) Neural basis of a perceptual decision in the parietal cortex (area LIP) of the rhesus monkey. J Neurophysiol 86: 1916-1936. Medline

Shulman GL, Ollinger JM, Akbudak E, Conturo TE, Snyder AZ, Petersen SE, Corbetta M (1999) Areas involved in encoding and applying directional expectations to moving objects. J Neurosci 19:9480-9496. Medline

Siegel M, Donner TH, Oostenveld R, Fries P, Engel AK (2007) Highfrequency activity in human visual cortex is modulated by visual motion strength. Cereb Cortex 17:732-741. CrossRef Medline

Siegel M, Engel AK, Donner TH (2011) Cortical network dynamics of perceptual decision-making in the human brain. Front Hum Neurosci 5:21. CrossRef Medline

Smith PL, Ratcliff R (2004) Psychology and neurobiology of simple decisions. Trends Neurosci 27:161-168. CrossRef Medline

Song JH, Nakayama K (2009) Hidden cognitive states revealed in choice reaching tasks. Trends Cogn Sci 13:360-366. CrossRef Medline

Summerfield C, Egner T (2009) Expectation (and attention) in visual cognition. Trends Cogn Sci 13:403-409. CrossRef Medline

Todorovic A, van Ede F, Maris E, de Lange FP (2011) Prior expectation mediates neural adaptation to repeated sounds in the auditory cortex: an MEG study. J Neurosci 31:9118-9123. CrossRef Medline

Tosoni A, Galati G, Romani GL, Corbetta M (2008) Sensory-motor mechanisms in human parietal cortex underlie arbitrary visual decisions. Nat Neurosci 11:1446-1453. CrossRef Medline

Usher M, McClelland JL (2001) The time course of perceptual choice: the leaky, competing accumulator model. Psychol Rev 108:550-592. CrossRef Medline

Wilke M, Logothetis NK, Leopold DA (2006) Local field potential reflects perceptual suppression in monkey visual cortex. Proc Natl Acad Sci U S A 103:17507-17512. CrossRef Medline

Williams ZM, Elfar JC, Eskandar EN, Toth LJ, Assad JA (2003) Parietal activity and the perceived direction of ambiguous apparent motion. Nat Neurosci 6:616-623. CrossRef Medline

Worden MS, Foxe JJ, Wang N, Simpson GV (2000) Anticipatory biasing of visuospatial attention indexed by retinotopically specific alpha-band electroencephalography increases over occipital cortex. J Neurosci 20:RC63. Medline

Yang T, Shadlen MN (2007) Probabilistic reasoning by neurons. Nature 447:1075-1080. CrossRef Medline 